\title{
A STONE-WEIERSTRASS THEOREM FOR SEMIGROUPS ${ }^{1}$
}

\author{
BY T. P. SRINIVASAN AND U. B. TEWARI
}

Communicated by Henry Helson, July 19, 1968

We prove here the following theorem. We shall assume throughout that the semigroups we consider are commutative and Hausdorff and that they have an identity (this last restriction can be relaxed in some places). By a semicharacter of a semigroup $G$ we shall mean a nonzero continuous homomorphism of $G$ into the set of complex numbers with absolute value less than or equal to 1 . The product of any two semicharacters of $G$ is not identically zero (since $G$ has an identity) and is therefore a semicharacter. For any semigroup $G$ we shall denote by $\hat{G}$ the set of all semicharacters of $G$ and by $G^{*}$ the subset of those semicharacters whose absolute value at any point is either 0 or 1 .

THEOREM. If $G$ is a compact semigroup such that $G^{*}$ separates the points of $G$, then any subset $A$ of $G^{*}$ which separates the points of $G$, which contains the unit semicharacter and which is closed under multiplication and under complex conjugation has to be the whole of $G^{*}$.

As immediate consequences we derive the known theorem on the duality of discrete semigroups which are unions of groups [1] and a theorem on the extendability of semicharacters on compact subsemigroups of a topological semigroup to the whole semigroup. Some other consequences of the theorem will be discussed elsewhere by us. We may remark that a similar theorem was announced as a footnote by Schwarz in [3] with $\hat{G}$ replacing $G^{*}$ in the above, but it must have been imprecisely stated because it seems to be false for the semigroup $G=[0,1]$ with the usual multiplication and usual topology.

PROOF OF THE ThEOREM. The set of all finite linear combinations of members of $A$ is a separating subalgebra closed under conjugation and containing constants, in the algebra $C(G)$ of all continuous complex valued functions on $G$ and is therefore uniformly dense in $C(G)$ by the Stone-Weierstrass theorem. Thus for every member $\alpha$ of $G^{*}$, there are distinct members $\alpha_{1}, \alpha_{2}, \cdots, \alpha_{k}$ in $A$ and complex constants $c_{1}, c_{2}, \cdots, c_{k}$ such that $\left\|\alpha-\sum_{i=1}^{k} c_{i} \alpha_{i}\right\|_{\infty}<1$. If $G$ were a group, a simple argument involving integration with respect to the normalized Haar measure on $G$ would show that $\alpha=\alpha_{i}$ for some $i$ and in particular, $\alpha \in A$. In the case of a semigroup $G$ we shall apply this argument

1 This research was supported by National Science Foundation Grant No. GP5585. 
to a suitable subgroup $G_{e}$ of $G$ to conclude that $\alpha=\alpha_{i}$ on $G_{e}$ for some $i$. Then an analysis of the generating idempotents of $G$ lying below the one which corresponds to $\alpha$ will complete the proof that $\alpha=\alpha_{i}$ on the whole of $G$.

Let $\alpha \in G^{*}$, and let $e=e(\alpha)$ be the generating idempotent corresponding to $\alpha$, that is, $e$ is the smallest idempotent in the compact subsemigroup $H=\{x:|\alpha(x)|=1\}$. We may remark that the complement $G \sim H$, in this case, is the vanishing ideal $N(\alpha)$ of $\alpha$, namely $G \sim H=\{x: \alpha(x)=0\}$ and the statement that the idempotent $e$ is generating means, for our purposes, that the semigroup $H$ (which is of course closed) is open in $G$, since the complement $G \sim H$ is closed. Let $G_{e}$ be the maximal group in $H$ containing $e$. It is well known that $H e \subset G_{e}$ and every semicharacter $\psi$ on $H$ with absolute value 1 everywhere is uniquely determined by its restriction $\phi$ to $G_{e}$ and in fact, $\psi(a)=\phi(a e)$ for all $a \in H$.

Choose distinct members $\alpha_{1}, \alpha_{2}, \cdots, \alpha_{k}$ in $A$ and complex numbers $c_{1}, c_{2}, \cdots, c_{k}$ such that $\left\|\alpha-\sum_{i=1}^{k} c_{i} \alpha_{i}\right\|_{\infty}<1$. If $d \lambda$ is the normalized Haar measure on $G_{e}$, then for any two characters $\psi, \eta$ of $G_{e}$ we have $\int \psi \bar{\eta} d \lambda=1$ or 0 according as $\psi=\eta$ or $\psi \neq \eta$. Hence, if $\alpha\left|G_{e} \neq \alpha_{i}\right| G_{e}$ for any $i$, then dropping those $i$ 's for which $\alpha_{i} \mid G_{\theta} \equiv 0$ and grouping together those $c_{i}$ 's for which $\alpha_{i} \mid G_{e}$ are identical, we shall have

$$
1>\int_{G_{e}}\left|\alpha-\sum_{i=1}^{k} c_{i} \alpha_{i}\right|{ }^{2} d \lambda=1+\Sigma\left|c_{i}\right|^{2},
$$

which is clearly impossible. Hence $\alpha$ coincides with $\alpha_{i}$ on $G_{e}$, for some $i$ and then by our earlier observation, $\alpha=\alpha_{i}$ on $H$. In other words, if $\alpha_{e}$ denotes the semicharacter which is equal to 1 on $H$ and is equal to 0 on $G \sim H$, then $\alpha=\alpha_{i} \alpha_{e}$. We shall show that $\alpha_{e} \in A$; it will follow that $\alpha$ itself belongs to $A$.

Suppose $\alpha_{e} \notin A$. In particular then, $\alpha_{e}$ is not the unit character and consequently $e$ is not the smallest idempotent in $G$. If $u$ is any idempotent of $G$ with $u<e$ (that is, $u e=u \neq e$ ), then there exists a member $\psi$ of $A$ which separates $u$ and $e$, and by replacing $\psi$ by $\psi \psi$, we may assume that $\psi$ takes only the values 0 and 1 . Then the idempotent $v=v(\psi)$ will satisfy $v<e$ and $v \$ u$. Thus for every idempotent $u$ in $G$ with $u<e$, there exists a generating idempotent $v=v(\psi)$ for some $\psi \in A$ such that $v<e$ and $v \quad$. This shows that if $E$ denotes the set of all generating idempotents $v<e$ such that $v=v(\psi)$ for some $\psi \in A$, then $e=\sup \{v: v \in E\}$. On the other hand, $E$ is directed for the relation $\leqq$ (since $A$ is closed under multiplication) and has the property that for every $u$ in $E$ there exists a $v$ in $E$ such that $v u$. An easy HeineBorel argument shows that an idempotent which is the supremum of 
any directed family of generating idempotents with the above property cannot be a generating idempotent. This contradiction shows that $\alpha_{\theta} \in A$.

CoROLlaRy 1. Let $G$ be a discrete semigroup which is a union of groups. Then $G$ is isomorphic to its second dual $\hat{G}^{\wedge}$ under the natural mapping which takes each point $x$ of $G$ to the evaluation map $\hat{x}^{\wedge}$ at $x$.

Proof. As is well known [2], a simple consideration of the idempotents of $G$ shows that the set $\hat{G}$ of all nonzero semicharacters of $G$ separates the points of $G$. This means that the map $x \rightarrow \hat{x}^{\wedge}$ is $1-1 . \hat{G}$ is a compact semigroup for the dual topology and the image of $G$ under the above map is a family $A$ of semicharacters on $\hat{G}$ satisfying the hypothesis of the main theorem. Therefore $A=(\hat{G})^{*}$. But since $G$ is a union of groups, so is $\hat{G}$ and in particular every semicharacter of $\hat{G}$ has absolute value either 0 or 1 at every point. Consequently, $A=(\hat{G})^{*}=\hat{G}^{\wedge}$. Since $\hat{G}$ is compact, $(\hat{G})^{*}$ and therefore also $\hat{G}^{\wedge}$ will be discrete in the dual topology $[1$, p. 247].

CoRollary 2. Let $G$ be any commutative topological semigroup such that $G^{*}$ separates the points of $G$. Then, if $H$ is any compact subsemigroup of $G$, every semicharacter of $H$ has an extension to a semicharacter of $G$.

Proof. The family of restrictions of the members of $G^{*}$ to $H$ coincides with $H^{*}$ by the main theorem. The proof will be complete if we show that every semicharacter of $H$ belongs to $H^{*}$. It suffices in turn to show that each point $x$ of $H$ belongs to some subgroup contained in $H$. But if $H_{x}=\left\{y \in H:|\alpha(y)|=|\alpha(x)|\right.$ for all $\left.\alpha \in H^{*}\right\}$ then $H_{x}$ is a closed subsemigroup of $H$ containing $x$, which is further cancellative because $H^{*}$ separates points; consequently $H_{x}$ is a group.

We state as a separate proposition the following fact contained in the concluding part of the above proof.

Proposition. If $H$ is a compact semigroup with identity such that $H^{*}$ separates the points of $H$, then $H$ is a union of groups, and consequently $\hat{H}=H^{*}$.

\section{REFERENCES}

1. C. W. Austin, Duality theorems for some commutative semigroups, Trans. Amer. Math. Soc. 109 (1963), 245-256.

2. E. Hewitt and H. S. Zuckerman, The $l_{1}$-algebra of a commutative semigroup, Trans. Amer. Math. Soc. 83 (1956), 70-97.

3. S. Schwarz, The theory of characters of commutative Hausdorff bicompact semigroups, Czechoslovak Math. J. (81) 6 (1956), 330-361.

Panjab University, India AND

University of California, Berkeley 